\title{
Large eddy simulation of boundary layer flow under cnoidal waves
}

\author{
Yin-Jun Li ${ }^{1}$ • Jiang-Bo Chen ${ }^{1}$ ・ Ji-Fu Zhou' ${ }^{1}$ • Qiang Zhang ${ }^{2}$
}

Received: 11 March 2015 / Revised: 17 May 2015 / Accepted: 6 July 2015 / Published online: 23 September 2015

(C) The Chinese Society of Theoretical and Applied Mechanics; Institute of Mechanics, Chinese Academy of Sciences and Springer-Verlag Berlin Heidelberg 2015

\begin{abstract}
Water waves in coastal areas are generally nonlinear, exhibiting asymmetric velocity profiles with different amplitudes of crest and trough. The behaviors of the boundary layer under asymmetric waves are of great significance for sediment transport in natural circumstances. While previous studies have mainly focused on linear or symmetric waves, asymmetric wave-induced flows remain unclear, particularly in the flow regime with high Reynolds numbers. Taking cnoidal wave as a typical example of asymmetric waves, we propose to use an infinite immersed plate oscillating cnoidally in its own plane in quiescent water to simulate asymmetric wave boundary layer. A large eddy simulation approach with Smagorinsky subgrid model is adopted to investigate the flow characteristics of the boundary layer. It is verified that the model well reproduces experimental and theoretical results. Then a series of numerical experiments are carried out to study the boundary layer beneath cnoidal waves from laminar to fully developed turbulent regimes at high Reynolds numbers, larger than ever studied before. Results of velocity profile, wall shear stress, friction coefficient, phase lead between velocity and wall shear stress, and the boundary layer thickness are obtained. The dependencies of these boundary layer properties on the asymmetric degree and Reynolds number are discussed in detail.
\end{abstract}

Ji-Fu Zhou

zhoujf@imech.ac.cn

$1 \quad$ Key Laboratory for Mechanics in Fluid Solid Coupling Systems, Institute of Mechanics, Chinese Academy of Sciences, Beijing 100190, China

2 School of Aerospace Engineering, Beijing Institute of Technology, Beijing 100081, China
Keywords Boundary layer structure - Turbulence - Large eddy simulation . Cnoidal wave

\section{Introduction}

Water wave-induced oscillatory boundary layer behaviors have long been the subject in the field of coastal engineering, owing to the profound physical implication for sediment transport [1-6]. Water waves are usually categorized by linearity. Linear water waves are sometimes called sinusoidal or harmonic waves in the literature, and they are a class of symmetric waves because their crest height is equal to their absolute trough height. Nonlinear waves are sometimes defined as asymmetric waves, typical examples of which are Stokes, cnoidal, and solitary waves. In shallow waters, linear/nonlinear waves result in harmonic/non-harmonic oscillatory boundary layers near the seabed.

To elucidate precisely wave boundary layer behaviors, regardless of symmetry, it is generally necessary to identify flow regimes, laminar or turbulent. According to the Reynolds number defined by $R_{\delta}=U_{0 m} \delta / \nu$, where $U_{0 m}$ is the amplitude of the free stream velocity, $\delta=\sqrt{2 v / \omega}$ is the Stokes thickness, $v$ is the kinematic viscosity of fluid, and $\omega$ is the angular frequency of wave motion, the wave boundary layer flow regime can be identified as follows: (1) the laminar regime if $R e_{\delta}<100$, (2) the disturbed laminar regime if $100<R e_{\delta}<550$, (3) the intermittent turbulent regime if $550<R e_{\delta}<3500$, (4) the fully developed turbulent regime if $\operatorname{Re}_{\delta}>3500$ [7-10].

Most previous researchers have mainly studied boundary layers under sinusoidal waves $[7,8,11,12]$. However, in shallow water circumstance of coastal areas, the wave 
profiles are generally asymmetric due to nonlinearity. A precise estimation of sediment transport under actual field conditions requires an adequate knowledge of boundary layer under asymmetric wave. Therefore, some experimental and theoretical studies have been carried out on boundary layer properties under asymmetric waves [1316].

With the pioneering work of Kondo [17], an analytical solution for laminar flow with an arbitrarily varying free-stream velocity has been derived by means of Laplace transformation. Since Kondo's solution is given in an integral form of elliptic function, it is inconvenient for calculation. Tanaka et al. [4] derived the velocity and wall shear stress in a Fourier series expansion, and it is helpful to study the laminar regime under asymmetric cnoidal waves.

Several experimental studies were performed by Nadaoka et al. $[18,19]$ and Ribberink and Al-Salem [20] on characteristics of asymmetric oscillatory boundary layers by using computer-controlled piston systems in a U-tube oscillating tunnel. Because of the complexity of previous U-tubes, Tanaka et al. [3,21] proposed a rather simple and inexpensive mechanism to simulate asymmetric cnoidal wave motion. Although a great many theoretical and experimental works have been devoted to the investigation of asymmetric boundary layer, they are generally limited to low values of Reynolds number $\left(R e_{\delta}<1500\right)$.

In addition, numerical simulations on this issue contribute to the high Reynolds number flow. In some works, Schlatter and Yamashita [22] and Spalart [23] used direct simulation to study the turbulent boundary layer. Jones and Launder's $k-\varepsilon$ model was used to predict the transitional behavior of the asymmetric oscillatory boundary layer by Tanaka et al. [3] and Sana et al. [24]. Moreover, another three versions of the two-equation model, namely the $k-\omega$ model by Wilcox [25], and the Baseline (BSL) and shear stress transport (SST) $k-\omega$ models by Menter [26], are employed to study asymmetric wave motion. Past studies showed that the BSL $k-\omega$ model is superior to other models [27]. However, two-equation models still focus on the disturbed laminar and the intermittent turbulent regimes. Moreover, these turbulent models are unable to give transient information of turbulent fluctuations.

During the last decade, large eddy simulation (LES) has been proved able to simulate accurately fully developed turbulent flows [11,28-31]. In their work, Lohmann et al. [30] used the classical Smagorinsky subgrid model to investigate a ventilated Stokes boundary layer in the turbulent regime at $R e_{\delta}=3464$ and obtained overall reasonable agreement with experimental data of Jensen et al. [8].

In the present paper, by using an infinite immersed plate oscillating in its own plane in quiescent water with a cnoidally varying velocity process, we simulate the boundary

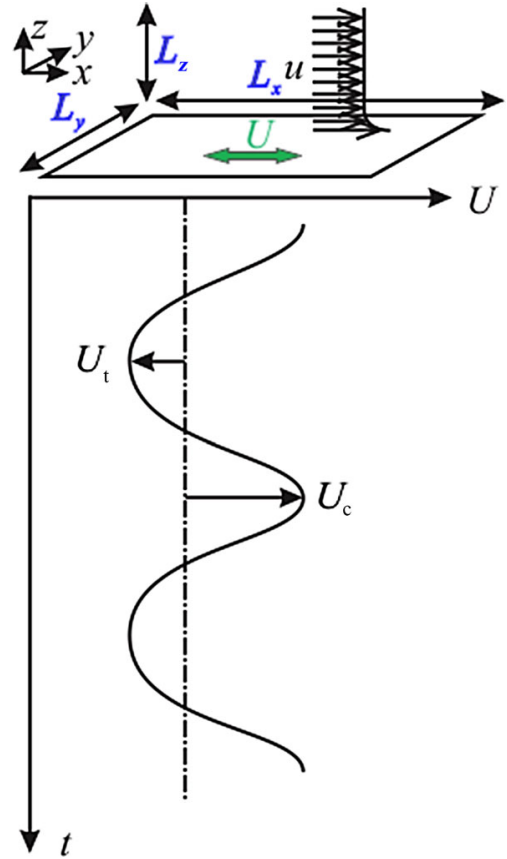

Fig. 1 Schematic description of the problem

layer under cnoidal waves as a typical case of asymmetric waves by means of LES with a Smagorinsky subgrid model. Particular attention is drawn to the variation of the asymmetric boundary layer flow field from laminar to fully developed turbulent regimes, where the Reynolds number is higher than ever studied before. The dependence of boundary layer properties, such as velocity profile, wall shear stress, friction coefficient, phase lead between velocity and wall shear stress, and the boundary layer thickness, on the asymmetric degree and Reynolds number are revealed.

\section{The problem formulation}

We use an infinite immersed plate oscillating in its own plane in quiescent water to simulate a water wave-induced boundary layer. As shown in Fig. 1, the coordinates are set by locating the origin at the oscillating plate with the $z$-axis normal to the plate and the $x$-axis parallel to the oscillating direction. Thus, $x, y$, and $z$ denote streamwise, spanwise, and vertical coordinates, respectively. The water takes the region of $z>0$.

In the non-inertial coordinate system, this flow can be equivalent to wave-induced flow by satisfying the flowing condition:

$$
\frac{\partial p}{\partial x}=-\rho \frac{\mathrm{d} U}{\mathrm{~d} t}, \frac{\partial p}{\partial y}=0, \frac{\partial p}{\partial z}=0
$$


Here, $p$ is the pressure of the wave flow field and $U$ is the oscillating velocity of the plate.

For asymmetric waves, it is necessary to define another dimensionless parameter besides the Reynolds number, i.e., the degree of wave asymmetry:

$$
A_{\mathrm{s}}=\frac{U_{\mathrm{c}}}{U_{\mathrm{c}}+U_{\mathrm{t}}}
$$

where $U_{\mathrm{c}}$ and $U_{\mathrm{t}}$ are the velocity magnitudes at wave crest and trough, respectively. Hence, we have $A_{\mathrm{s}}=0.5$ for symmetric waves. If $U_{\mathrm{c}}$ is kept unchanged, a larger $A_{\mathrm{s}}$ means a smaller $U_{\mathrm{t}}$. According to the first-order cnoidal wave theory, $A_{\mathrm{S}}$ can be expressed as $(1-E / K) / m^{2}$, a single-valued function of elliptic modulus $m$, where $K=\int_{0}^{\frac{\pi}{2}} \frac{\mathrm{d} \theta}{\sqrt{1-m^{2} \sin ^{2} \theta}}$ and $E=\int_{0}^{\frac{\pi}{2}} \sqrt{1-m^{2} \sin ^{2} \theta} \cdot \mathrm{d} \theta$ are the complete integrals of the elliptic function of the first and the second kind, respectively.

Reynolds number $R e_{\delta}$ defined by Stokes layer thickness as below,

$\operatorname{Re}_{\delta}=\frac{U_{\mathrm{c}} \delta}{v}$

is used to discuss the flow regime.

The computational model is sketched in Fig. 1. The flow is induced by a periodically oscillating bottom plate, which results in a cnoidal variation of the free-stream velocity,

$U=\frac{U_{\mathrm{c}}}{1-\overline{c n^{2}}}\left\{c n^{2}\left(\frac{2 K t}{T}\right)-\overline{c n^{2}}\right\}$

Here, $c n$ denotes Jacobi's elliptic function. The cnoidal wave becomes sinusoidal if $m=0$ or solitary if $m=1 ; t$ denotes time and $T$ the period of oscillations; the overbar "-" denotes time average over a wave cycle.

In their paper, Tanaka et al. [4] derived the expressions of flow velocity $u$ and wall shear stress $\tau_{0}$ of laminar boundary layer under cnoidal waves as follows,

$$
\begin{aligned}
& u=\frac{U_{\mathrm{c}}}{B_{N}} \sum_{n=1}^{N} a_{n}\left[\cos (n \omega t)-\mathrm{e}^{-\beta_{n} z} \cos \left(n \omega t-\beta_{n} z\right)\right] \\
& \tau_{0}=\rho U_{\mathrm{c}} \sqrt{\omega \nu} \frac{1}{B_{N}} \sum_{n=1}^{N} \sqrt{n} a_{n} \cos \left(n \omega t+\frac{\pi}{4}\right)
\end{aligned}
$$

where $\beta_{n}=\sqrt{n \omega /(2 \nu)}, B_{N}$ and $a_{n}$ are coefficients obtained from the series expressing of elliptic function. By introducing dimensionless parameters $u^{*}=u / U_{\mathrm{c}}, t^{*}=\omega t, z^{*}=z / \delta$, and $\tau_{0}^{*}=\tau_{0} /\left(\rho U_{\mathrm{c}} \sqrt{\omega \nu}\right)$, the dimensionless velocity and wall shear stress can be obtained. Equations (5) and (6) are the laminar theory solution used in the following sections.

\subsection{The mathematical model}

The continuity equation and the spatially filtered timedependent 3-D incompressible Navier-Stokes equations used for LES are given by

$\frac{\partial \overline{u_{i}}}{\partial x_{i}}=0$

$\frac{\partial \overline{u_{i}}}{\partial t}+\frac{\partial \overline{u_{i} u_{j}}}{\partial x_{j}}=-\frac{1}{\rho} \frac{\partial \bar{p}}{\partial x_{i}}+v \frac{\partial^{2} \overline{u_{i}}}{\partial x_{j} \partial x_{j}}+\overline{f_{i}}$.

Here, the overline "-" denotes filtering at a scale $\Delta, x_{i}$ denote the coordinates of Cartesian system with the corresponding velocity component $u_{i}$ and the corresponding mass force component $\overline{f_{i}}$. Incidentally, $\partial \bar{p} / \partial x_{i}$ denotes the pressure gradient under equivalent cnoidal wave, calculated by the above Eqs. (1) and (4). According to $\overline{u_{i} u_{j}}=$ $\overline{u_{i} u_{j}}+\left(\overline{u_{i} u_{j}}-\overline{u_{i} u_{j}}\right)$, neglecting mass force $\overline{f_{i}}$, Eq. (8) yields

$\frac{\partial \overline{u_{i}}}{\partial t}+\frac{\partial \overline{u_{i} u_{j}}}{\partial x_{j}}=-\frac{1}{\rho} \frac{\partial \bar{p}}{\partial x_{i}}+v \frac{\partial^{2} \overline{u_{i}}}{\partial x_{j} \partial x_{j}}+\frac{\partial\left(\overline{u_{i} u_{j}}-\overline{u_{i} u_{j}}\right)}{\partial x_{j}}$

In LES, the unresolved scale is taken into consideration by additional shear stress $\tau_{\mathrm{sgs}, i j}=\overline{u_{i} u_{j}}-\overline{u_{i} u_{j}}$, of which the off-diagonal part is modeled by an eddy viscosity concept, and in which the subscript "sgs" indicates the subgrid part of variables. The Smagorinsky model for $\tau_{\mathrm{sgs}, i j}$ defined as

$\tau_{\mathrm{sgs}, i j}=-2 v_{\mathrm{sgs}} \overline{S_{i j}}$,

is used, where $v_{\mathrm{sgs}}$ is the eddy viscosity and $\overline{S_{i j}}$ is the resolved strain rate tensor. They are defined as

$\overline{S_{i j}}=\frac{1}{2}\left(\frac{\partial \overline{u_{i}}}{\partial x_{j}}+\frac{\partial \overline{u_{j}}}{\partial x_{i}}\right)$,

$v_{\mathrm{sgs}}=\left(C_{\mathrm{s}} \Delta\right)^{2}|\bar{S}|$.

Here, $C_{\mathrm{S}}$ is the Smagorinsky coefficient, $\Delta$ is the filter scale that depends on the grid size, and $|\bar{S}|=\sqrt{2 \overline{S_{i j} S_{i j}}}$ is the modulus of the strain rate. In the present paper, $C_{\mathrm{s}}=0.1$ is found to be most appropriate, based on comparison with experimental data.

The variables in the problem are non-dimensionalized as follows:

$$
\begin{aligned}
& t^{*}=\omega t, \quad x_{i}^{*}=\frac{x_{i}}{\delta}, \quad u_{i}^{*}=\frac{u_{i}}{U_{\mathrm{c}}} \\
& \boldsymbol{u}^{*}=\left(u_{1}^{*}, u_{2}^{*}, u_{3}^{*}\right), \quad p^{*}=\frac{p}{\rho U_{\mathrm{c}}^{2}} .
\end{aligned}
$$



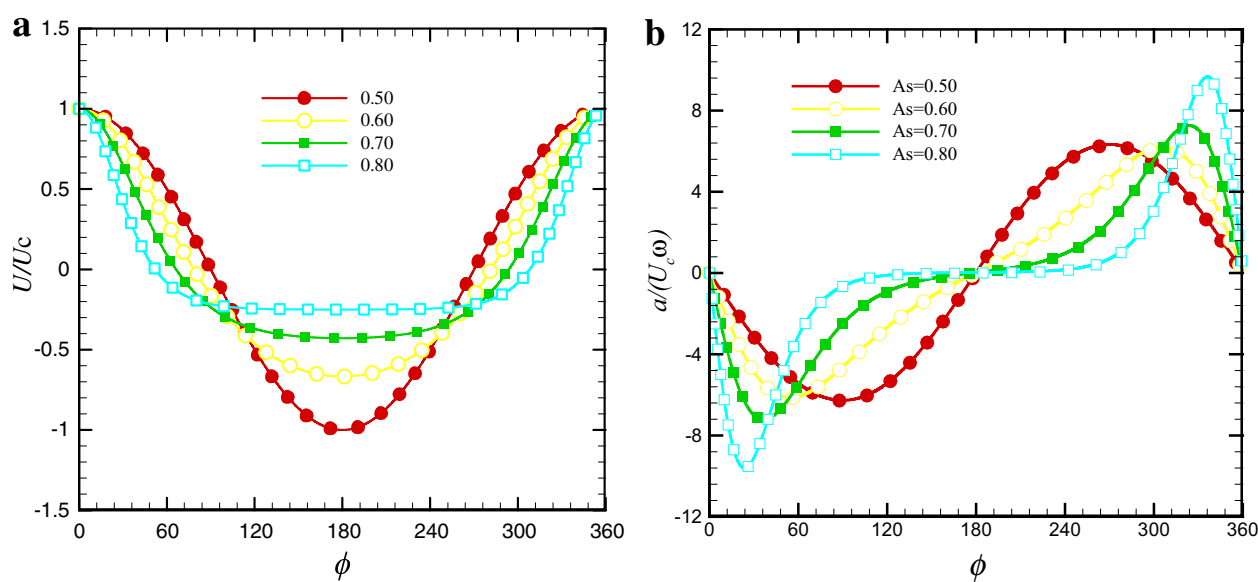

Fig. 2 Free-stream velocity $\mathbf{a}$ and acceleration $\mathbf{b}$ for cnoidal waves of different $A_{\mathrm{S}}\left(A_{\mathrm{S}}=0.50,0.60,0.70,0.80\right)$

The resulting non-dimensional forms of the governing equations are,

$$
\frac{\partial u^{*}}{\partial t}+\frac{R e_{\delta}}{2}\left(u^{*} \cdot \nabla\right) u^{*}=-\frac{R e_{\delta}}{2} \nabla p^{*}+\frac{1}{2} \nabla^{2} u^{*}
$$

$\nabla \cdot \boldsymbol{u}^{*}=0$.

Since turbulence is homogeneous in the streamwise and spanwise directions, periodic boundary conditions are adopted. In the vertical direction, a non-slip boundary condition is enforced at the bottom wall and a free-slip condition at the top boundary.

\subsection{Numerical method}

Uniform grids are adopted in the streamwise and spanwise directions, and non-uniform staggered grids are used in the vertical direction. All cases of computational grids adopt $64 \times 64 \times 128$ nodes, which satisfy calculation convergence condition and the precision requirement as pointed by Piomelli [32]: the spacing in streamwise and spanwise directions are respectively $\Delta x^{+}=50-150$ and $\Delta y^{+}=15-40$; and the location of the first-layer grid in the vertical direction is $z^{+} \leqslant 1.0$, where the superscript + denotes dimensionless parameter normalized by $v / u_{\tau \mathrm{c}}$ and $u_{\tau \mathrm{c}}=\sqrt{\tau_{\mathrm{c}} / \rho}$. The intensive degree of non-uniform staggered grids can be adjusted by intensive parameter $\alpha$ from Qiang [33].

The simulations use a mixed spectral and finite difference algorithm. Derivatives in the streamwise and spanwise directions are treated with a pseudo-spectral method, while derivatives in the vertical direction are computed with second-order center difference in the vertical staggered grids. The second-order Adams-Bashforth method is adopted for time-marching.

\section{Results and discussion}

In this section, we first validate the model with the experimental and theoretical data and then apply the LES model to explore the characteristics of turbulent boundary layer at high Reynolds number.

For the convenience of comparison with symmetric wave, the degree of cnoidal wave asymmetry $A_{\mathrm{s}}$ is set to be 0.50 , $0.60,0.70$, and 0.80 , respectively. Figure 2 shows the velocity profile and acceleration profile of cnoidal waves for different $A_{\mathrm{s}}$. The free stream flow reversal occurs roughly at the phases of $90^{\circ}, 78^{\circ}, 66^{\circ}, 52^{\circ}$ in the first half-cycle and $270^{\circ}, 282^{\circ}, 294^{\circ}, 308^{\circ}$ in the second half-cycle, respectively, for different $A_{\mathrm{s}}$ from 0.50 to 0.80 . Additionally, in the first half-cycle, the profile of corresponding acceleration is with the more steep front and higher (absolute) acceleration value when $A_{\mathrm{s}}$ increases from 0.50 to 0.80 , but it goes in the opposite direction in the other half-cycle.

\subsection{Velocity field}

Figures 3 and 4 show the comparison of the profiles of the mean velocity between the numerical data and the theoretical solution in the laminar regime $\left(R e_{\delta}=100\right)$ expressed by Eq. (5). It is observed that the numerical results exhibit an excellent agreement with the theoretical ones both for symmetric wave ( $A_{\mathrm{s}}=0.50$, Fig. 3$)$, and asymmetric wave $\left(A_{\mathrm{s}}=0.70\right.$, Fig. 4$)$. The velocity overshoots are also well predicted. In addition, the velocity profiles of the first and second half-periods are symmetric with respect to the line $u / U=0$ for symmetric wave in laminar flow, while those of the asymmetric wave are asymmetric.

In the disturbed laminar flow regime, the present LES model also performs well. Figure 5 compares the numerical results with the experimental data from Sana et al.'s [24] Case 1, where $R e_{\delta}=392$ and $A_{\mathrm{s}}=0.69$. In spite of some 


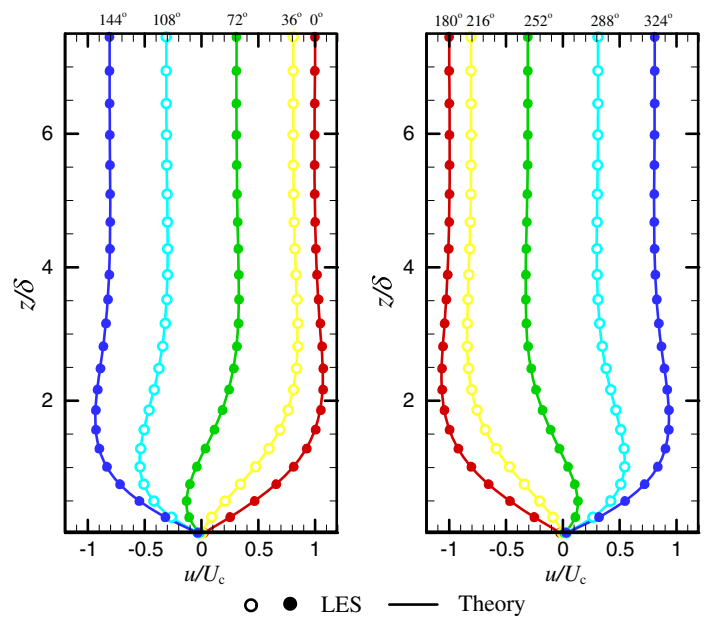

Fig. 3 Comparison of simulated (dots or circles) and theoretical (solid curves) non-dimensional ensemble-averaged velocity profiles (streamwise component) at different phases in one cycle for $A_{\mathrm{s}}=0.50, R e_{\delta}=$ 100

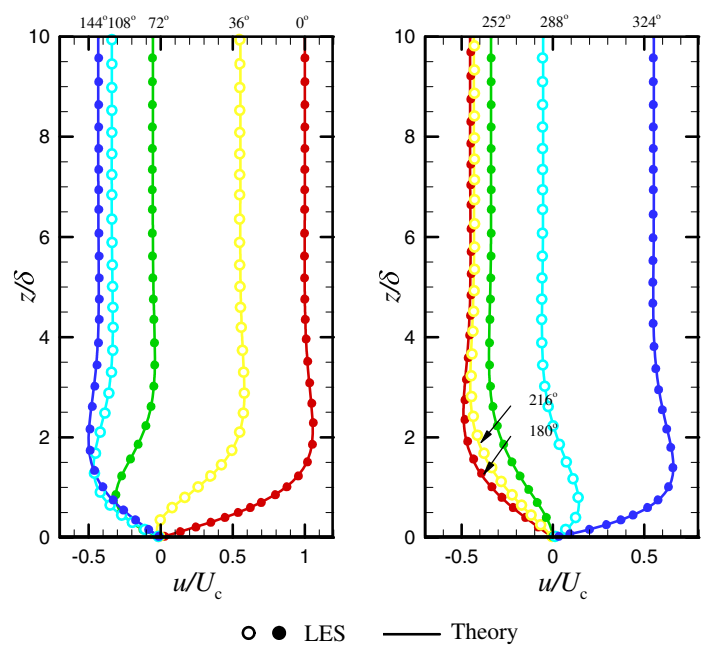

Fig. 4 Comparison of simulated (dots or circles) and theoretical (solid curves) non-dimensional ensemble-averaged velocity profiles (streamwise component) at different phases in one cycle for $A_{\mathrm{s}}=0.70, R e_{\delta}=$ 100

discrepancies at the phase of $252^{\circ}, 288^{\circ}$, and $324^{\circ}$, the overall agreement is satisfactory. Particularly, the agreement in the area very near the bottom is perfect, which implies that the bed shear stress is well modeled. Figure 6 illustrates a very good agreement between the present numerical and the theoretical data expressed by Eq. (5). These indicate that the current LES method is accurate enough in simulating disturbed laminar flow.

When the flow is in the intermittent turbulent flow regime, there are slight discrepancies between the present numerical and the experimental data as shown in Fig. $7\left(R e_{\delta}=\right.$ $\left.936, A_{\mathrm{s}}=0.62\right)$. The main discrepancies occur at the phase of $36^{\circ}, 72^{\circ}, 108^{\circ}, 252^{\circ}$, and $288^{\circ}$ near the wall, which may be partly due to the free-stream velocity reversal at $75^{\circ}$ and $285^{\circ}$, respectively, partly due to the experimental errors aris-

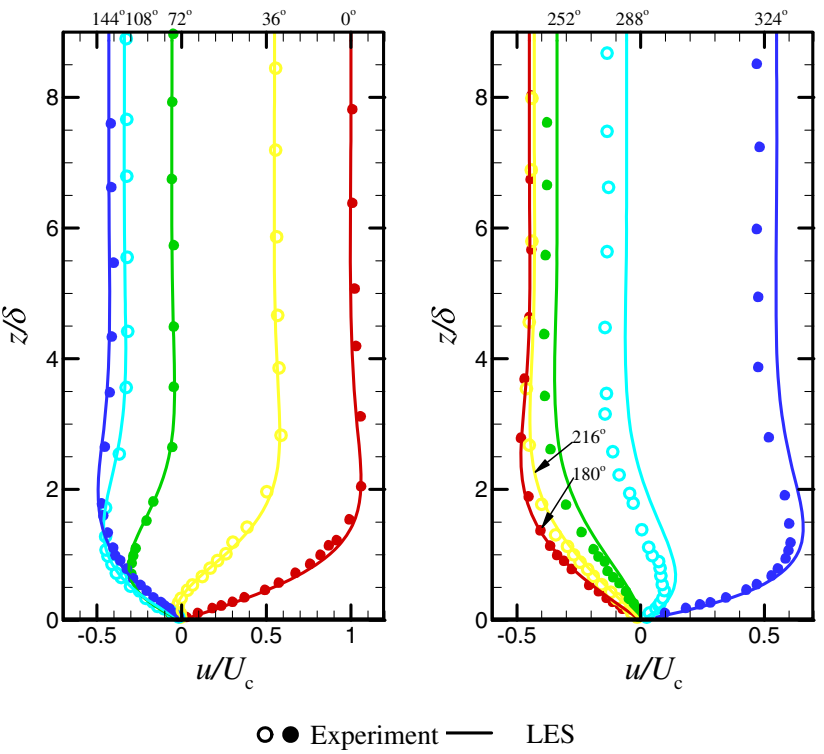

Fig. 5 Comparison of simulated (solid curves) and experimental (dots or circles) non-dimensional ensemble-averaged velocity profiles (streamwise component) at different phases in one cycle for $A_{\mathrm{s}}=0.69, R e_{\delta}=392$. Experimental data is from Sana et al.'s [24] Case 1

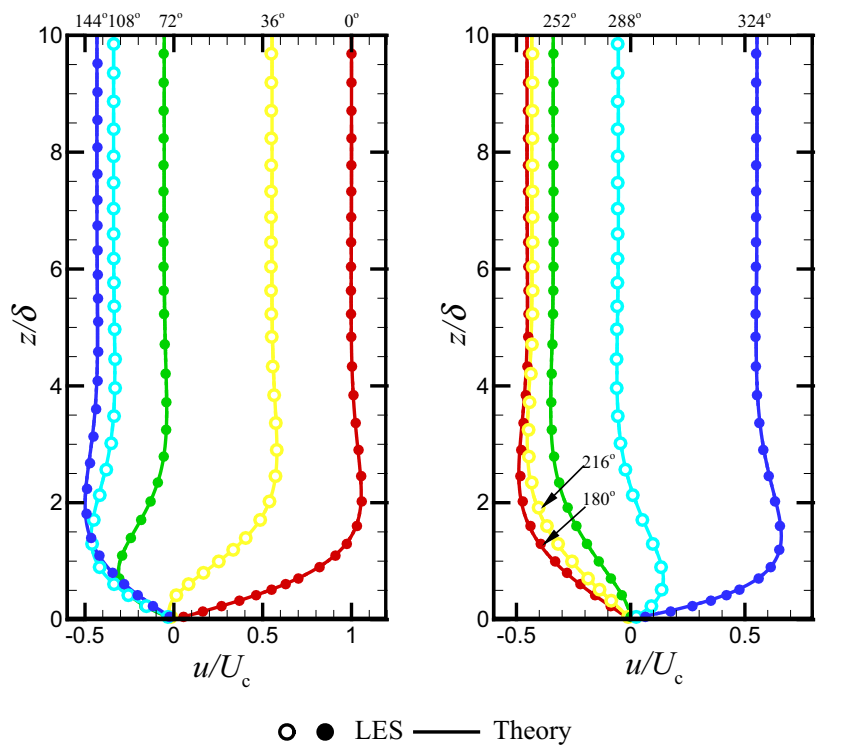

Fig. 6 Comparison of simulated (dots or circles) and theoretical (solid curves) non-dimensional ensemble-averaged velocity profiles (streamwise component) at different phases in one cycle for $A_{\mathrm{s}}=0.69, \operatorname{Re}_{\delta}=$ 392

ing from the measuring difficulty in the near bottom region. And the discrepancies at the phase of 36 are of insignificance, especially in the intermittent turbulent regime.

In the latest studies, the fully developed turbulent flow of asymmetric wave could not be achieved. Thus, we use the symmetric wave data instead to verify the present LES model in the fully developed turbulent regime. Figure 8 from 


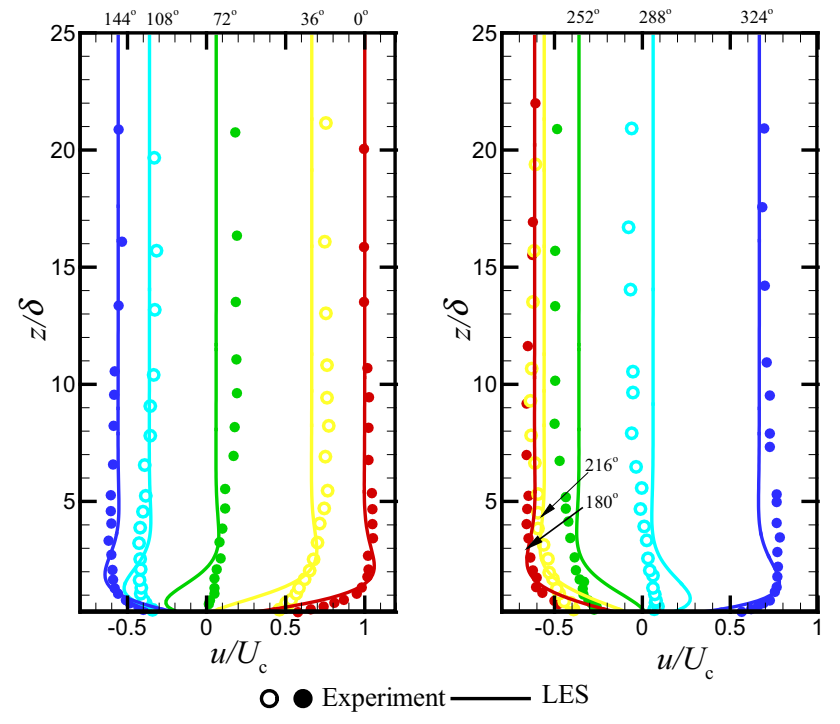

Fig. 7 Comparison of simulated (solid curves) and experimental (dots or circles) non-dimensional ensemble-averaged velocity profiles (streamwise component) at different phases in one cycle for $A_{\mathrm{s}}=$ $0.62, R e_{\delta}=936$. Experimental data is from Tanaka et al.'s [4] Case $\mathrm{N} 02$

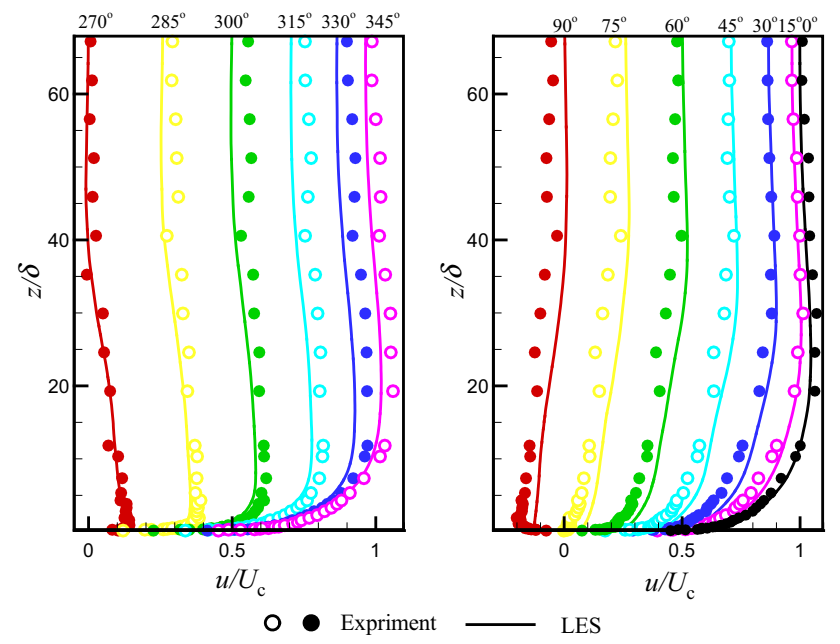

Fig. 8 Comparison of simulated (solid curves) and experimental (dots or circles) non-dimensional ensemble-averaged velocity profiles (streamwise component) at different phases in one cycle for $A_{\mathrm{s}}=$ $0.50, \operatorname{Re}_{\delta}=3464$. Experimental data is from Jensen et al.'s [8] Test 10

Qiang [33] shows the comparison of the profiles of the mean velocity between the simulation of the same methodology as the current paper and Jensen et al.'s [8] Test 10 data, where $R e_{\delta}=3464$ and $A_{\mathrm{s}}=0.50$. Although the present numerical results are slightly smaller at the phase of $270^{\circ}-345^{\circ}$ and slightly larger at the phase of $0^{\circ}-90^{\circ}$ than the experimental data, the simulations satisfactorily match the experimental results in the fully developed turbulent flow with acceptable errors. More importantly, the agreement in the area very near the bottom is perfect, which implies that the bed shear stress is well modeled in the fully developed turbulent flow.

From Figs. 3 to 8, we can conclude with confidence that the present LES model is accurate and effective enough, except for the transitional flow regime, which is marginal for the present study.

Figure 9 presents the velocity profiles affected by Reynolds number. Because of flow transition, the curves are clearly categorized by $R e_{\delta}=1000$ at each phase. If we change $A_{\mathrm{s}}$, the same phenomenon occurs. Furthermore, due to the reversal phase of $78^{\circ}$ for $A_{\mathrm{s}}=0.60$, there exist velocity profiles with opposite flow directions at different vertical locations near the phase of $80^{\circ}$.

Figure 10 shows the effects of $A_{\mathrm{S}}$ and $R e_{\delta}$ on velocity profiles. The difference mainly exists near the wall and lasts from laminar to turbulent flow through the whole cycle. The amplitude of velocity at $A_{\mathrm{s}}=0.50$ is always maximum. With $R e_{\delta}$ increasing, the boundary layer thickness normalized by Stokes layer thickness becomes larger, which will be detailed in Sect. 3.3.

It is worth emphasizing that the properties of the fully developed turbulent asymmetric boundary layers have not been accounted for in most of the previous literature due to difficulties in numerical computation and laboratory measurement. Figure 11 presents the stream velocity in semi-log plot near the wall, where $\phi=0^{\circ}-180^{\circ}, R e_{\delta}=4000$. The dash and the dash-dot straight lines demonstrate logarithmic law of streamwise velocity distribution of two directions in the case of symmetric waves. All velocity profiles for different $A_{\mathrm{S}}$ at different phases are found to be approximately linear lines, but with different slopes and intercepts in semi$\log$ coordinates. The velocity profiles of symmetric waves follow the conventional logarithmic law of turbulent boundary layers, but those of asymmetric waves do not. In addition, we also verify that when $A_{\mathrm{S}}$ changes from $0.52,0.55$ to 0.58 , the corresponding velocity profiles vary from the profile for $A_{\mathrm{s}}=0.50$ to that for $A_{\mathrm{s}}=0.60$ gradually.

Figure 12 shows time series of the streamwise velocity at different locations away from the wall. The degrees of asymmetry of the fitted lines are about 0.60 , being attributed to the fact that the flow is driven by the cnoidal wave with asymmetric degree of 0.60 . At the location of $z / \delta=20$, there exists velocity overshoot.

Figure 13 indicates that the degree of asymmetry $A_{\mathrm{S}}$ has a great effect on streamwise velocity. The fitted lines are very similar to the respective free-stream velocity processes. By comparing velocity fluctuations between cases of different $A_{\mathrm{s}}$, it is found that the main difference exists at the trough, i.e. the larger $A_{\mathrm{S}}$ is, the smaller velocity fluctuations are. This is owing to the different valley values of free-stream velocity. Figure 14 shows the same difference of the fluctuations of the spanwise and vertical velocities. From these two figures, the fluctuations occur in 

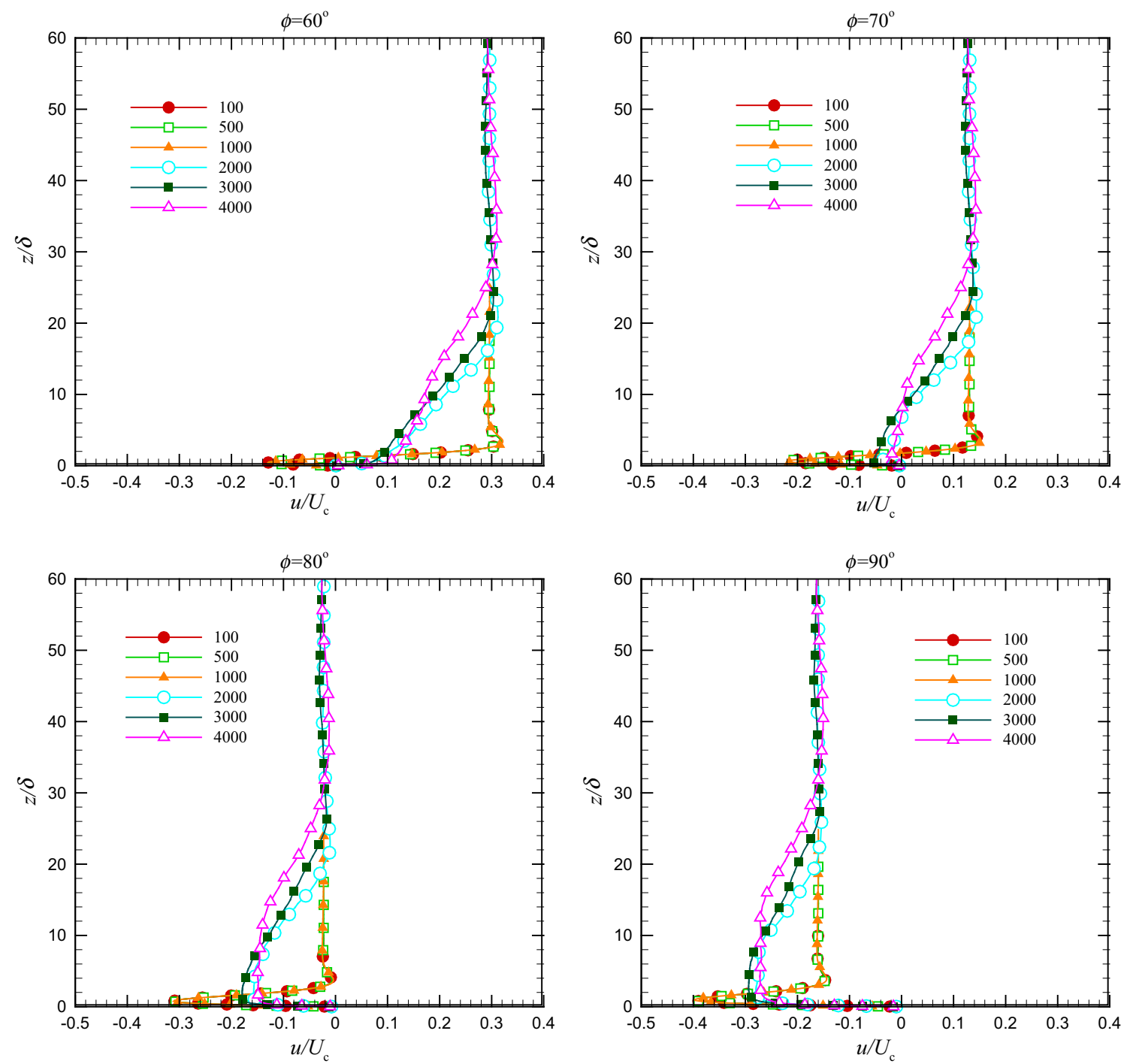

Fig. 9 Non-dimensional ensemble-averaged velocity profiles (streamwise component) at different phases in one cycle for $A_{\mathrm{s}}=0.60, R e_{\delta}=$ $100-4000$

three velocity components, suggesting prevailing of turbulence. Furthermore, they exist obviously through the whole cycle, implying that the flow is in fully developed turbulent regime.

\subsection{Wall shear stress}

Figures 15 and 16 indicate that the present LES results of wall shear stress agree well with the theoretical solution of laminar flow. The wall shear stress of asymmetric wave is asymmetric in laminar flow, while that of the symmetric wave is symmetric.

Figure 17 shows that the numerical wall shear stress matches the theoretical and the experimental data very well in the disturbed laminar regime. But when the flow is in the intermittent turbulent regime, there exists a certain phase difference as shown in Fig. 18. Nonetheless, the present numerical result of the amplitude seems better than that of $k-\varepsilon$ model [24]. The mismatch may be attributed to the inaccuracy of the experimental data, which is obtained using a momentum integral instead of direct measurement. Likewise, we use the wall shear stress of the symmetric wave to verify the LES method in the fully developed turbulent regime. Figure 19 indicates that the present LES method is fairly accurate in simulating wall shear stress in the fully developed regime. The numerical results satisfactorily are consistent with the experimental data collected by Jensen et al.'s [8] Test 10.

Figure 20 depicts the variation of wall shear stress at different Reynolds numbers. The wall shear stress is distinct from laminar to turbulent regimes. The trough phase of the non-dimensional normalized wall shear stress occurs earlier $50^{\circ}$ in laminar flow than in turbulent flow, and crest phase 

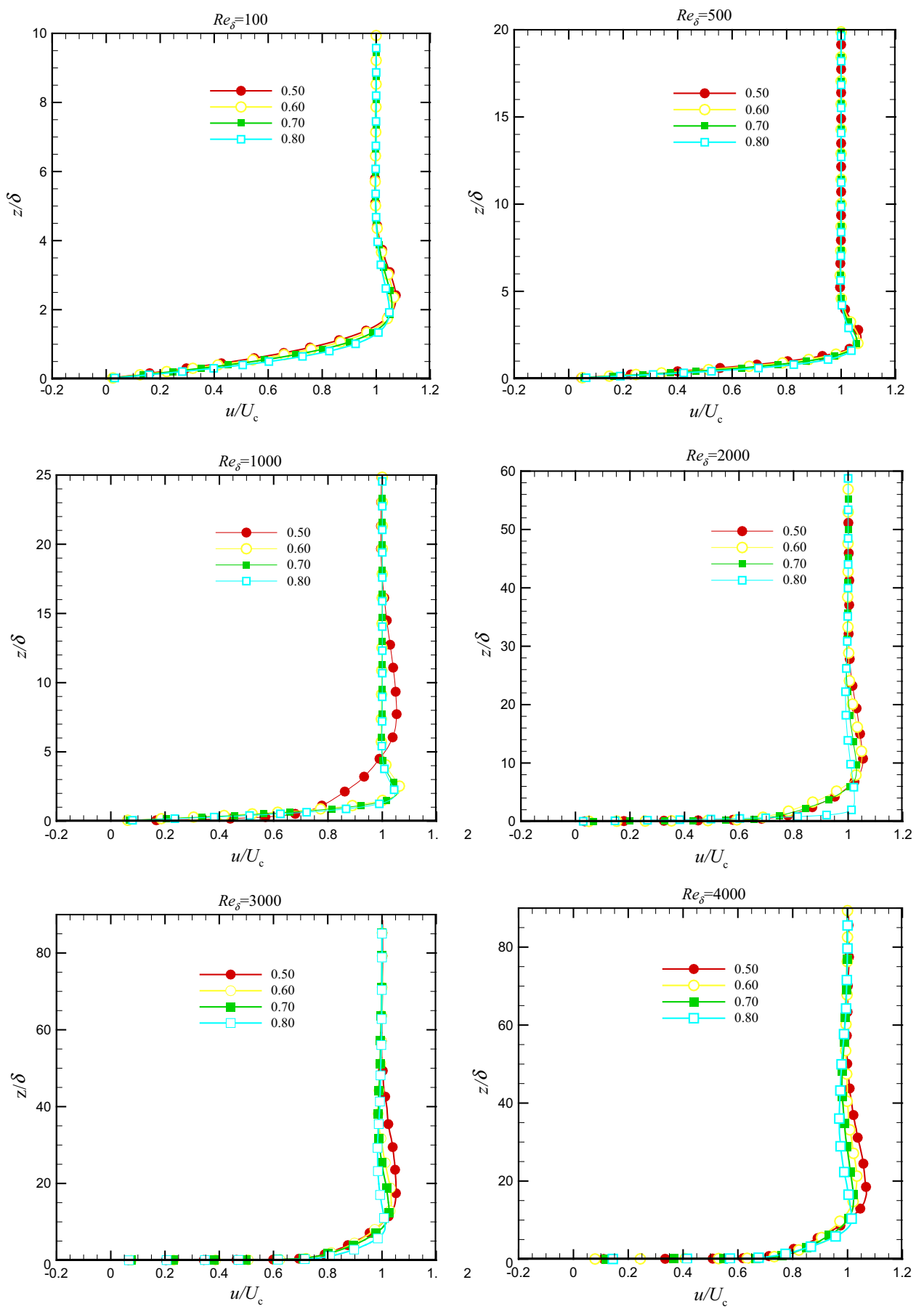

Fig. 10 Non-dimensional ensemble-averaged velocity profiles (streamwise component) at different Reynolds number in one cycle for $\phi=0^{\circ}, A_{\mathrm{s}}=$ $0.50-0.80, R_{\delta}=100,500,1000,2000,3000,4000$

earlier $25^{\circ}$. When $R e_{\delta}$ changes from 1000 to 2000 (that is, the flow regime is in the intermittent turbulent regime), the wall shear stress history behaves very much different, which has much to do with the instability of transitional behavior of the boundary layer. In addition, when $R e_{\delta}<1000$ or $R e_{\delta}>2000$, the effect of $R e_{\delta}$ on wall shear stress seems very weak.
Figure 21 shows normalized wall shear stresses for $A_{\mathrm{s}}=$ $0.50-0.80$ at $R e_{\delta}=4000$, which is similar to the history of free stream velocity. The amplitude of trough gets smaller with $A_{\mathrm{s}}$ increasing, and the trough occurs earlier accordingly.

The phase lead between wall shear stress and free-stream velocity plays an important role in unsteady sediment motion. For asymmetric cases, it is necessary to distinguish the phase 

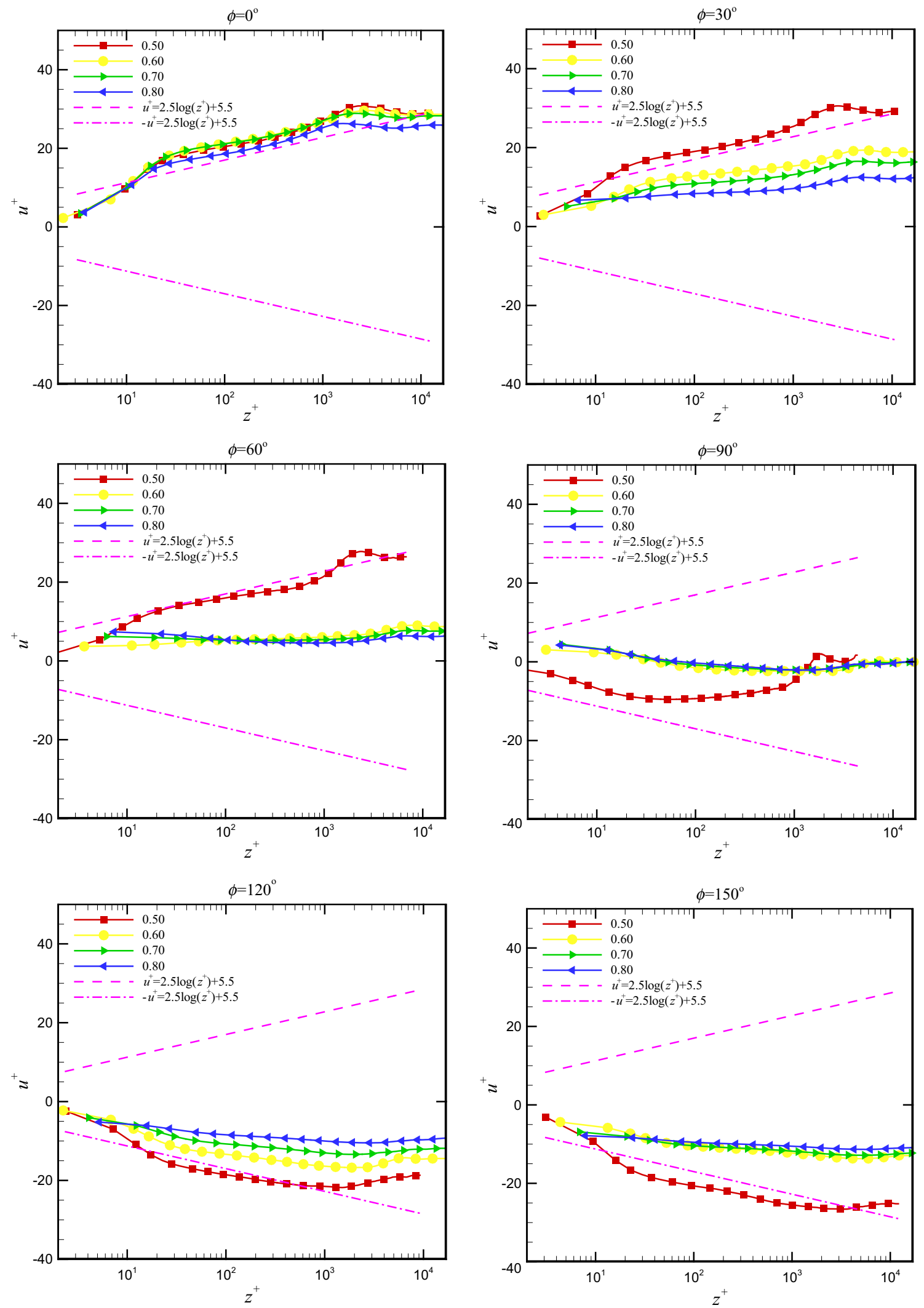

Fig. 11 Non-dimensional ensemble-averaged of the streamwise velocity for $\phi=0^{\circ}-180^{\circ}, A_{\mathrm{s}}=0.50-0.80, R e_{\delta}=4000$ 


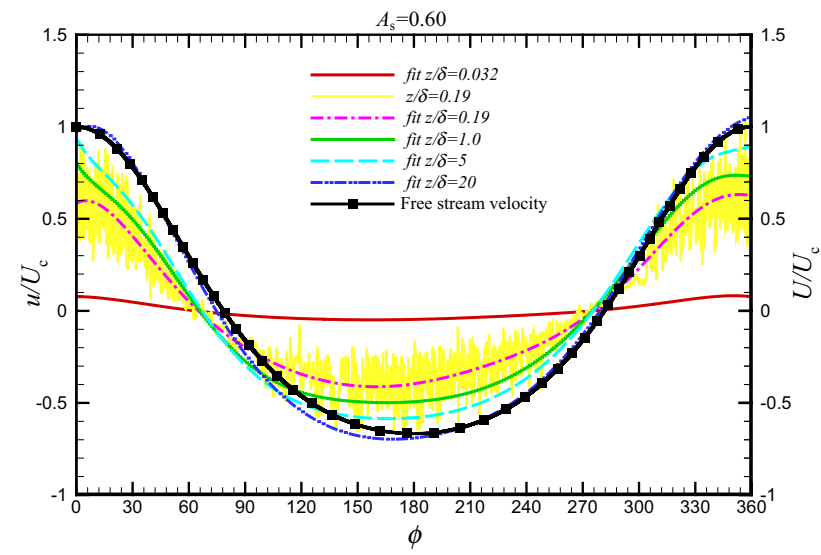

Fig. 12 Non-dimensional streamwise velocity for $A_{\mathrm{s}}=0.60, R e_{\delta}=$ 4000 . Only the free stream velocity curve refers to the right hand vertical axis. All the fitted lines are 10th-degree polynomials

lead at the crest and trough. Here, $\Delta \phi_{\mathrm{c}}$ is defined as the phase lead at the crest and $\Delta \phi_{\mathrm{t}}$ at the trough.

Figure 22 shows that the phase lead of symmetric wave for laminar flow is $\Delta \phi=45^{\circ}$, which agrees exactly with the theoretical value. Further studies of the phase lead under asymmetric cnoidal waves imply following points (see Fig. 23). In general, the phase lead $\Delta \phi_{\mathrm{c}}$ and $\Delta \phi_{\mathrm{t}}$ with different $A_{\mathrm{s}}$ remain unchanged respectively in laminar regime,
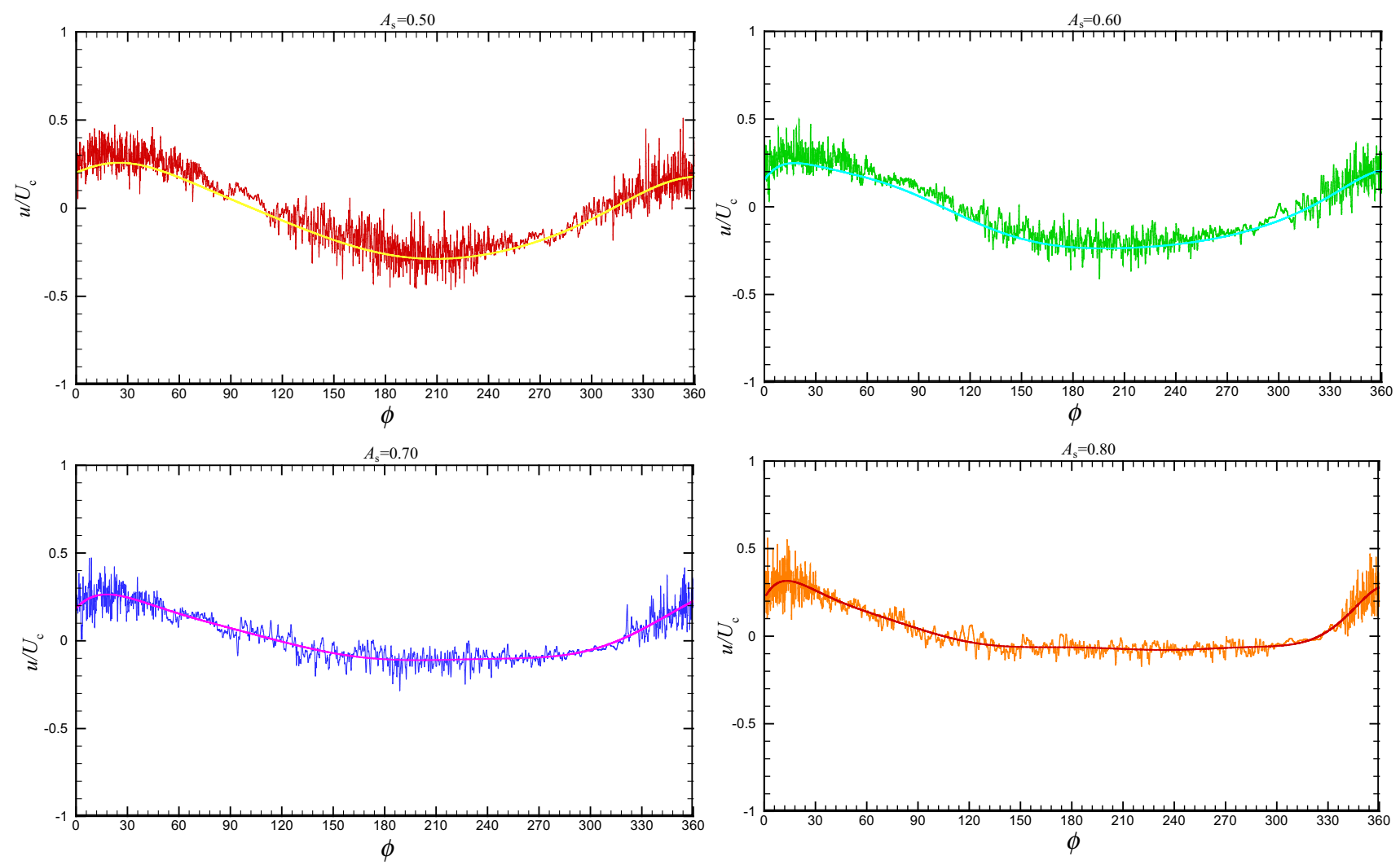

Fig. 13 Non-dimensional streamwise velocity for $A_{\mathrm{s}}=0.50-0.80, R e_{\delta}=4000, z / \delta=1$. The center lines are fitted by 10 th-degree polynomials 

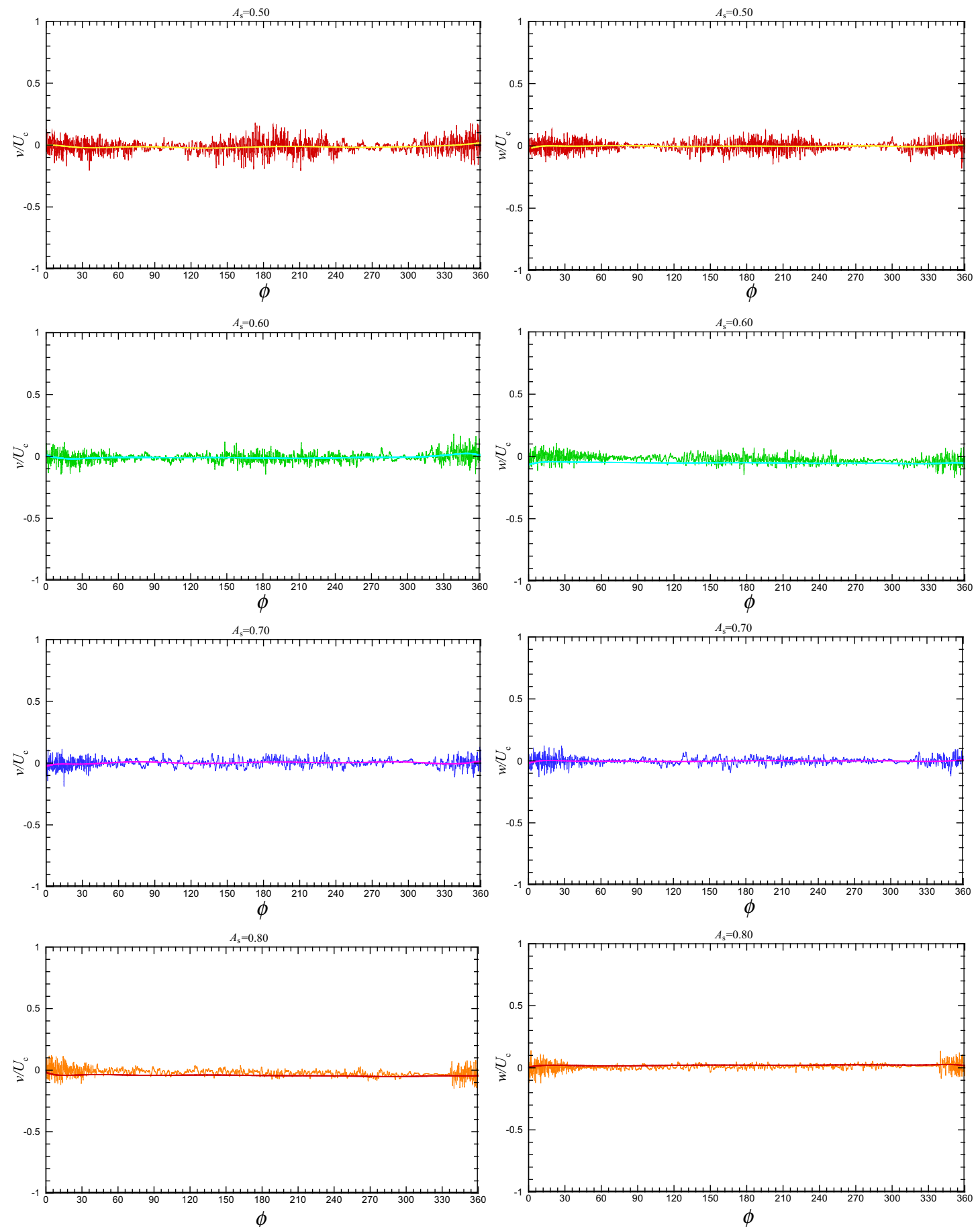

Fig. 14 Non-dimensional spanwise (left column) and vertical velocity (right column) for $A_{\mathrm{s}}=0.50-0.80, e_{\delta}=4000, z / \delta=1$. The center lines are fitted by 10th-degree polynomials

$f_{w \mathrm{t}}=\frac{\tau_{0(\mathrm{t})}}{\frac{1}{2} \rho U_{\mathrm{c}}^{2}}$.

Here, $\tau_{0(\mathrm{c})}$ and $\tau_{0(\mathrm{t})}$ are the maximum shear stress during the period of wave crest and wave trough respectively.

According to Jensen et al.'s [8] research, the flow regime can be divided by friction factor in sinusoidal wave bound- ary layers. It is seen from Fig. 24 that there is a general agreement between the present LES results and the experimental results pertaining to the friction factor for symmetric waves. Figure 25 depicts the variation of the friction factor at the trough with Reynolds number for asymmetric waves. In the log-log plot, from the fitting result, the wave friction 


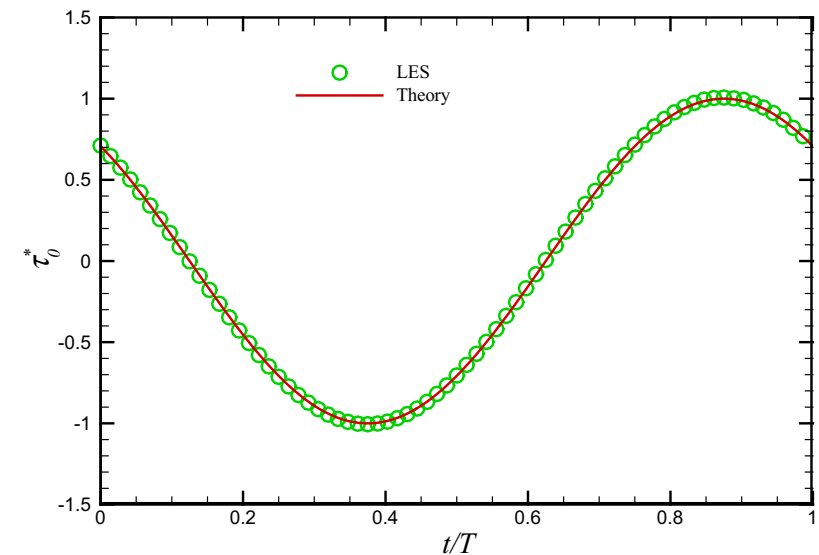

Fig. 15 Comparison of simulated (circles) and theoretical (solid curve) non-dimensional wall shear stress for $A_{\mathrm{s}}=0.50, R_{\delta}=100$

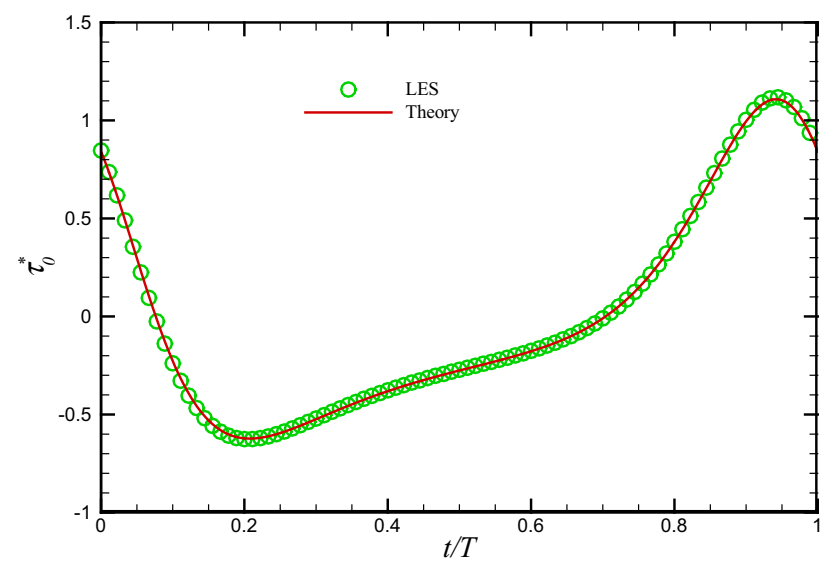

Fig. 16 Comparison of simulated (circles) and theoretical (solid curve) non-dimensional wall shear stress for $A_{\mathrm{s}}=0.70, \operatorname{Re}_{\delta}=100$

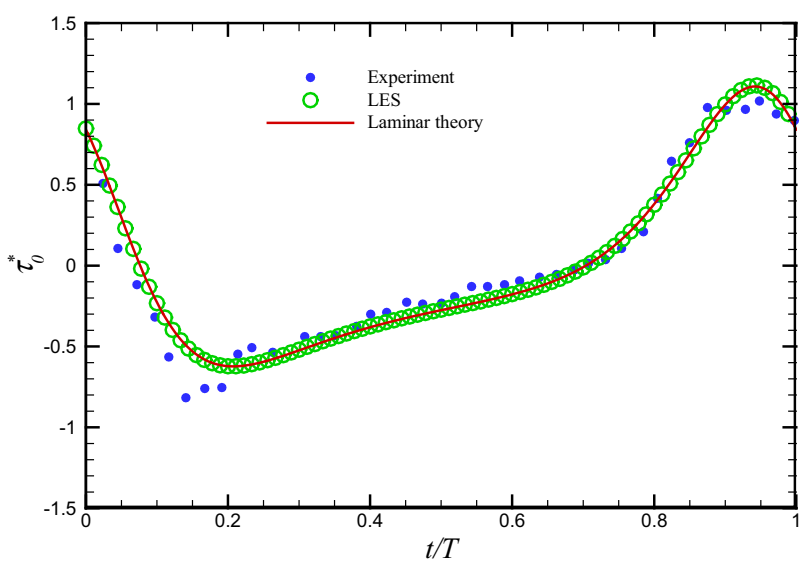

Fig. 17 Comparison of simulated (circles), experimental (dots) and theoretical (solid curve) non-dimensional wall shear stress for $A_{\mathrm{s}}=$ 0.69, $R e_{\delta}=392$. Experimental data is from Sana et al.'s [24] Case 1

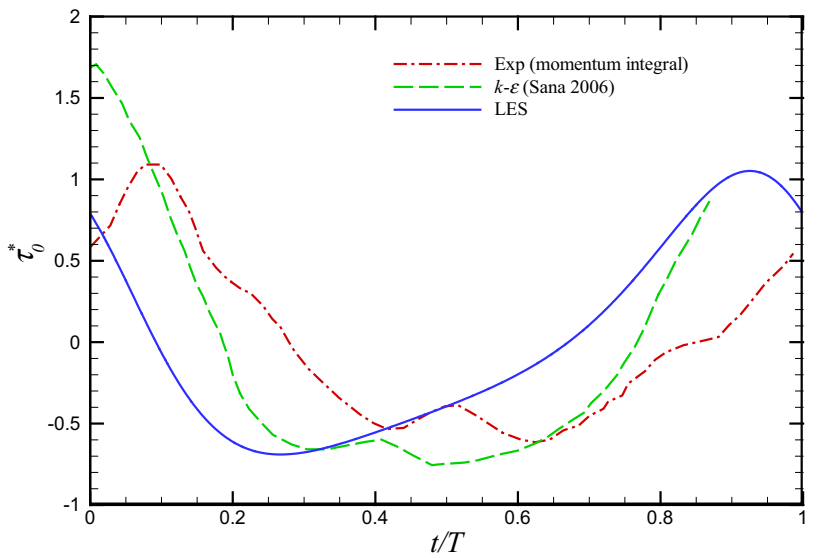

Fig. 18 Comparison of non-dimensional wall shear stress of LES, $k-\varepsilon$ model and experiment for $A_{\mathrm{s}}=0.62, R e_{\delta}=936$. Experimental data is from Sana et al.'s [24] Case N02

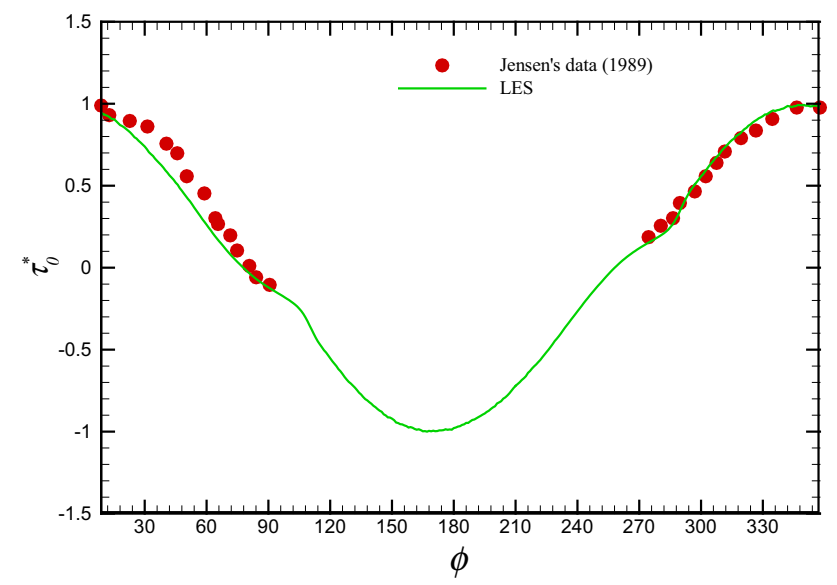

Fig. 19 Comparison of simulated (solid curve) and experimental (dots) non-dimensional wall shear stress for $A_{\mathrm{s}}=0.50, R e_{\delta}=3464$. Experimental data is from Jensen et al.'s [8] Test 10

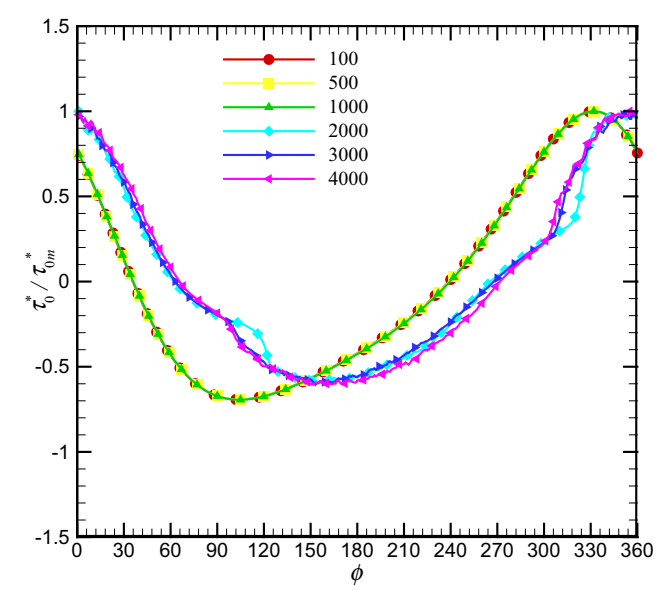

Fig. 20 Non-dimensional normalized wall shear stress at different Reynolds number in one cycle for $A_{\mathrm{s}}=0.60, R e_{\delta}=100-4000$ 


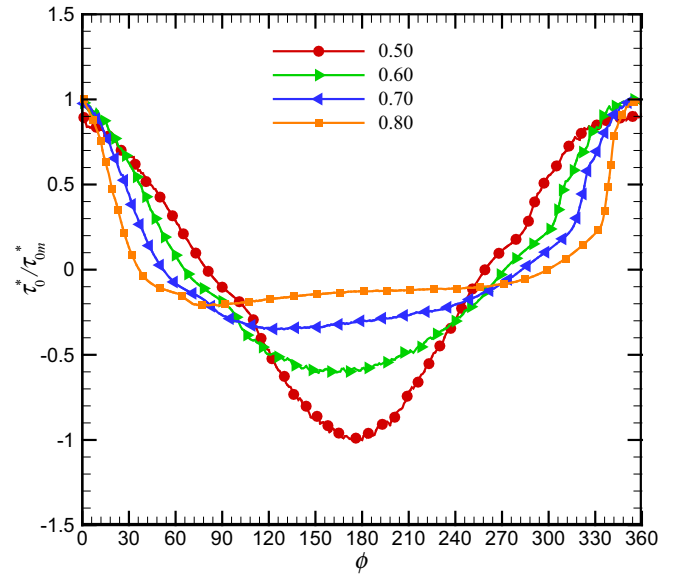

Fig. 21 Non-dimensional normalized wall shear stress in one cycle for $A_{\mathrm{S}}=0.50-0.80, \operatorname{Re}_{\delta}=4000$

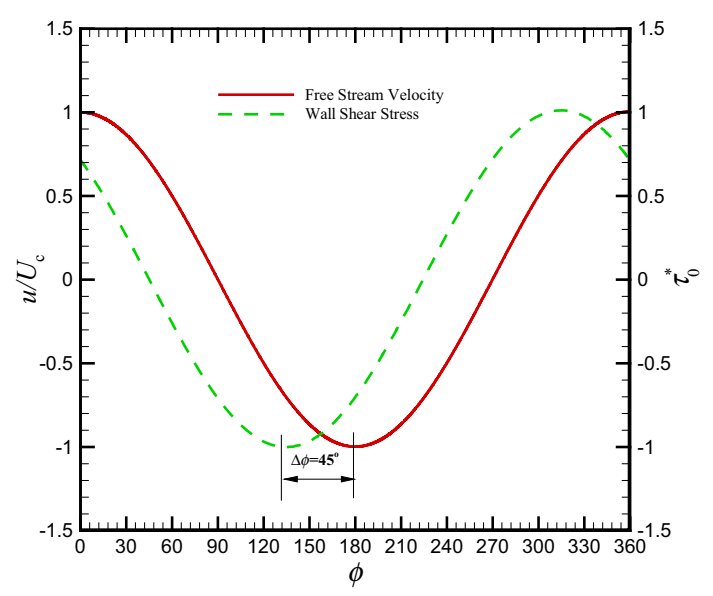

Fig. 22 Phase lead between wall shear stress and free-stream velocity for $A_{\mathrm{s}}=0.50, \operatorname{Re}_{\delta}=100$

factor decreases approximately linear decreasing in laminar regime, grows parabolically in transitional regime and gradually decreases to a stable value in turbulent regime against Reynolds number. As $A_{\mathrm{s}}$ gets larger, the friction factor at the trough becomes smaller. In addition, the critical $R e_{\delta}$ from laminar to turbulent regime gets larger with $A_{\mathrm{s}}$ increasing.

\subsection{Boundary layer thickness}

Figures 26 and 27 present the non-dimensional boundary layer thickness $\delta_{\mathrm{BL}} / \delta$ plotted against $R e_{\delta}$, respectively for symmetric and asymmetric waves. The $\delta_{\mathrm{BL}}$ defined by Jensen et al. [8] is adopted. The agreement among the numerical, experimental, and theoretical data from Fig. 24 in Jensen et al. [8] is quite good in Fig. 26. Figure 27 shows the effect of $A_{\mathrm{s}}$ on $\delta_{\mathrm{BL}} / \delta$. It is observed that the non-dimensional boundary layer thickness does not feel the effect of asymmetry in the laminar flow regime, but it does in the turbulent regime, decreasing with $A_{\mathrm{s}}$ increasing.

\section{Conclusion}

In the present paper, we have proposed to use an infinite immersed plate executing a cnoidal oscillation in its own plane in quiescent water to simulate the cnoidal wave boundary layer. A large eddy simulation approach with Smagorinsky subgrid model is adopted to investigate the relationship between the flow characteristics of cnoidal wave boundary layers and two important parameters, namely the degree of wave asymmetry and the Reynolds number, especially in the fully developed turbulent flow regime. Conclusions of the cnoidal wave boundary layer behaviors different from those of symmetric wave boundary layers are drawn as follows:

(1) The large-eddy simulation approach with Smagorinsky subgrid model can efficiently predict the mean properties of cnoidal wave boundary layers in different flow regimes, such as velocity field, wall shear stress, friction coefficient, phase lead, and boundary layer thickness. Although some discrepancies are observed in the cases of transitional flow, the statistics are accurate enough in laminar and fully developed turbulent regimes. 

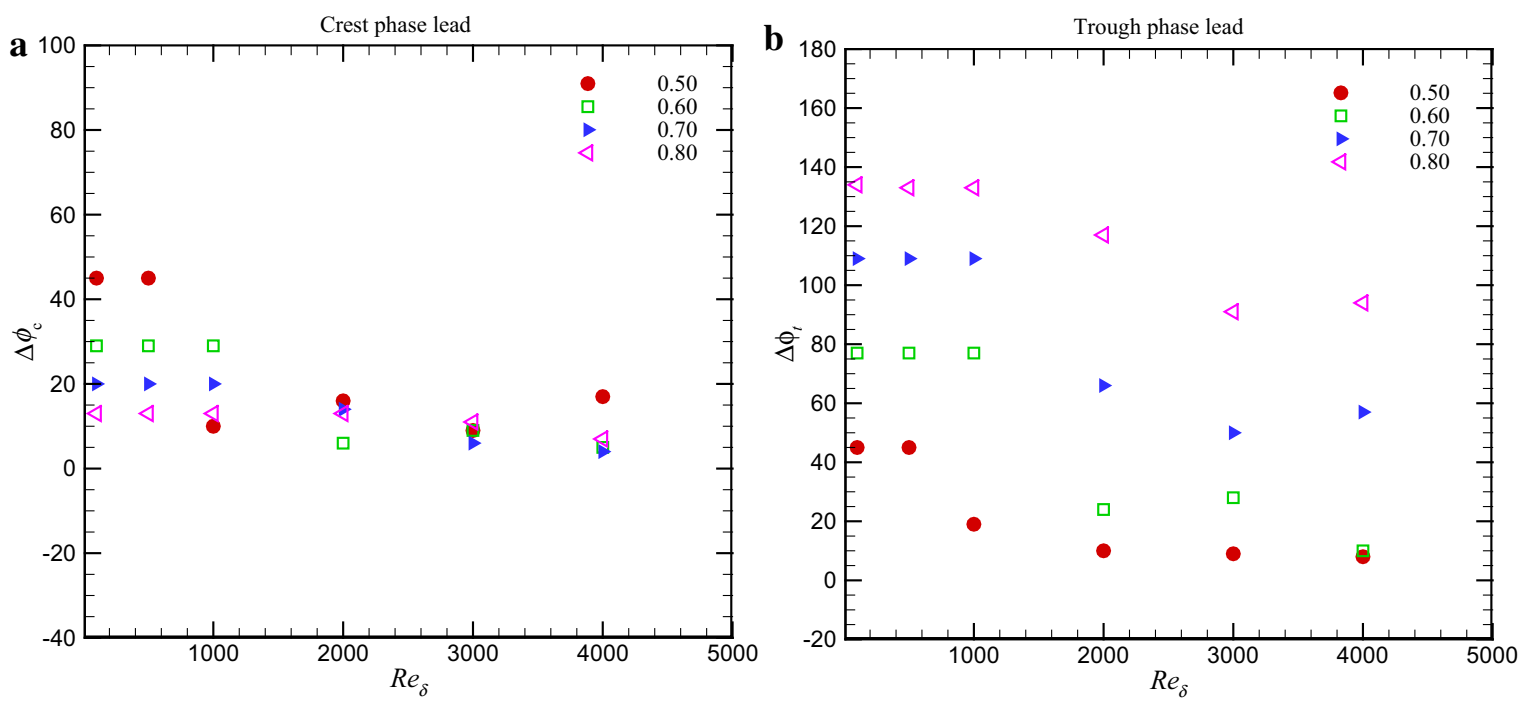

Fig. 23 Phase lead between wall shear stress and free-stream velocity versus $R e_{\delta}$ for $A_{\mathrm{s}}=0.50-0.80$. a Crest phase lead. b Trough phase lead

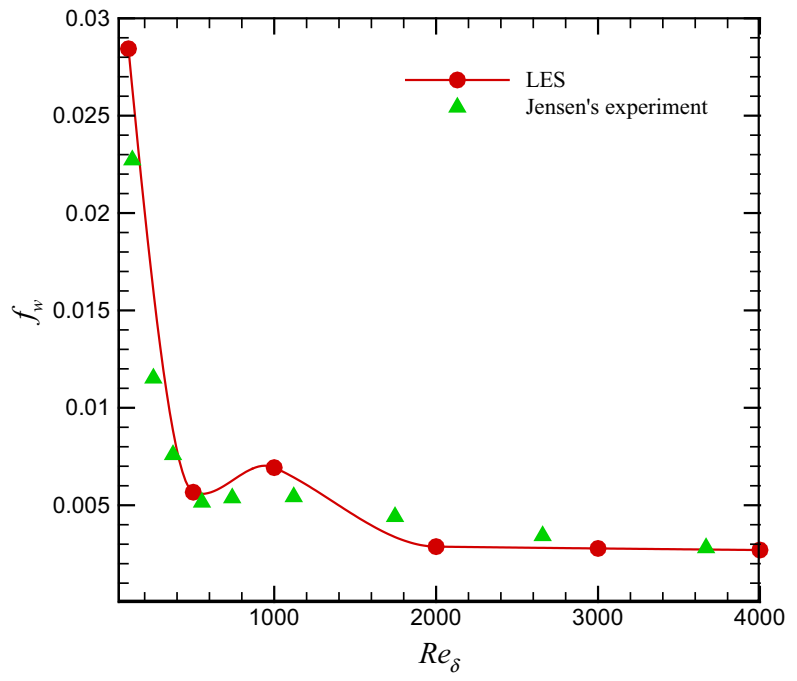

Fig. 24 Wave friction factor for symmetric wave versus $R e_{\delta}$, Experimental data from Jensen et al. [8]. The curve of LES result is fitted by Akima spline

(2) The degree of wave asymmetry plays a significant role in the boundary layer structures. In the fully developed turbulent regime, the absolute value of velocity valley decreases with the degree of asymmetry increasing. But all velocity profiles for different asymmetry degree approximately presents logarithmic distribution. And the velocity profiles of symmetric waves follow the conventional logarithmic law of turbulent boundary layers, but those of asymmetric waves do not.

(3) The wall shear stress process is obviously distinct from laminar to turbulent regimes. Its phase lead to velocity process behaves differently in three flow regimes. It keeps invariant in laminar regime, gets smaller with

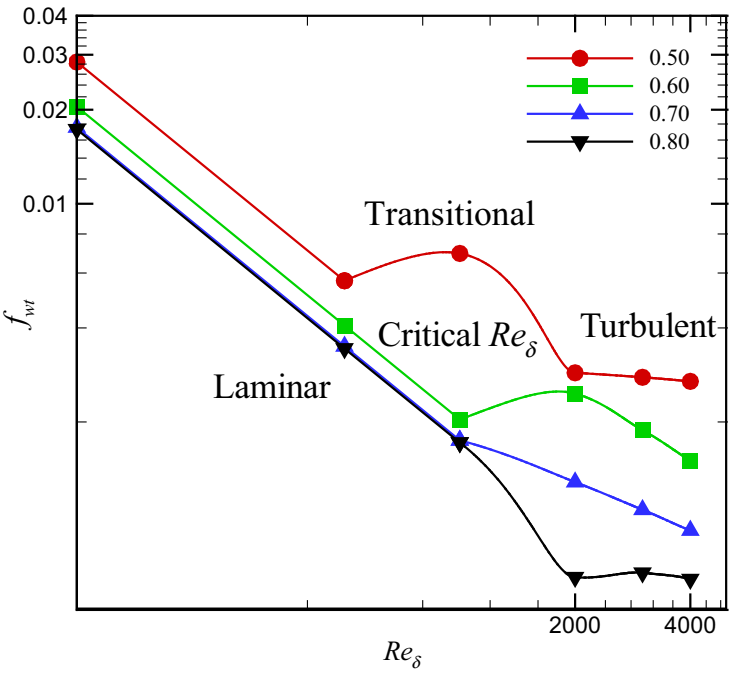

Fig. 25 Wave friction factor for asymmetric wave with different $A_{s}$ versus $\operatorname{Re}_{\delta}\left(A_{\mathrm{S}}=0.50-0.80\right)$. All the above curves are fitted by Akima spline

increase of Reynolds number in the transitional regime, and tends to another constant in the turbulent regime. And in turbulence, the variation of wall shear stress with the degree of asymmetry resembles that of corresponding velocity. The trough phase lead increases with the degree of asymmetry in laminar and turbulent regimes, while the crest one decreases in the laminar regime, but keeps almost unchanged in the turbulent regime.

(4) The wave friction factor in the laminar regime linearly decreases in double logarithmic coordinates, grows parabolically in the transitional regime and decreases gradually in the turbulent regime against Reynolds number. As the degree of asymmetry gets larger, the friction factor at the trough becomes smaller. The flow regime 


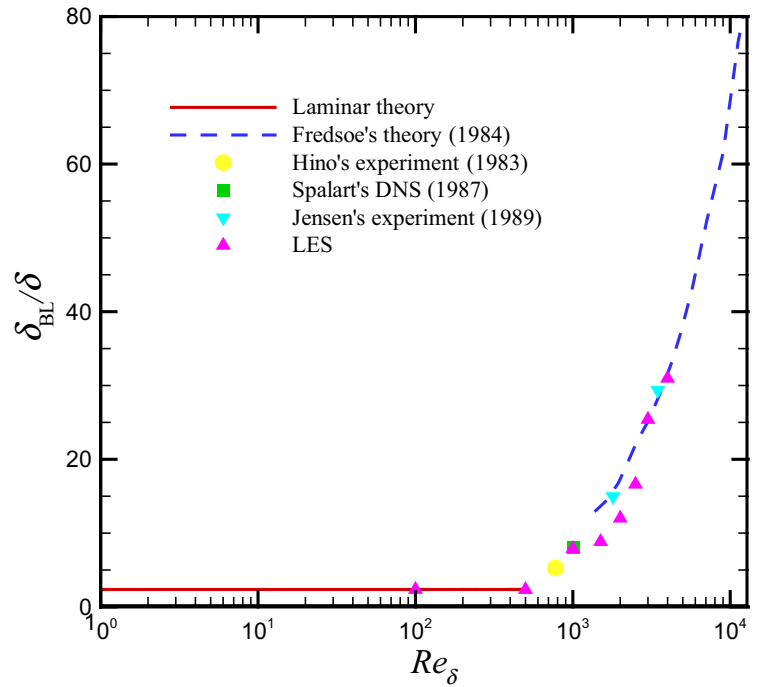

Fig. 26 Non-dimensional boundary layer thickness versus $R e_{\delta}$

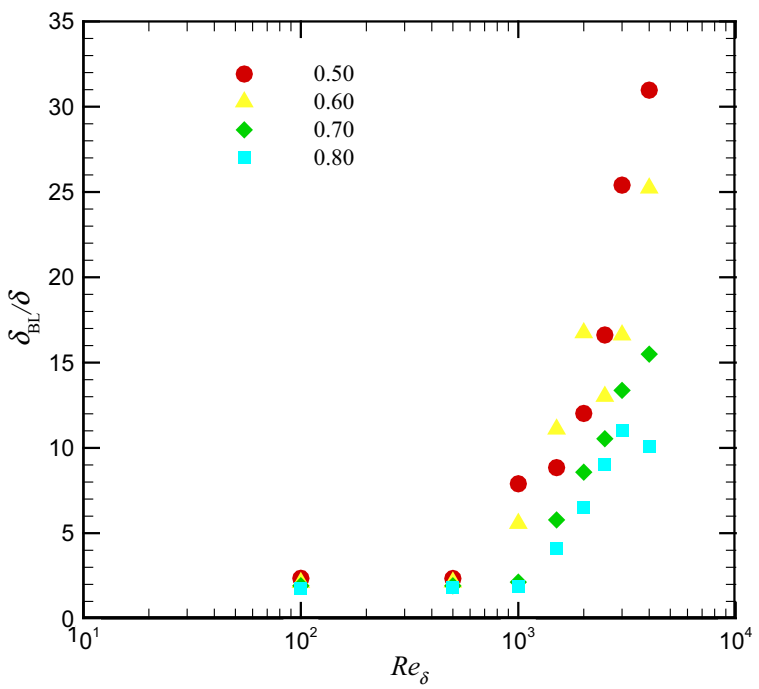

Fig. 27 Non-dimensional boundary layer thickness versus $R e_{\delta}$ for $A_{\mathrm{s}}=0.50-0.80$

can be identified by the variation of friction factor, and the critical Reynolds number from laminar to turbulent regimes increases with the degree of asymmetry. In addition, the boundary layer thickness increases with the degree of asymmetry.

Acknowledgments We very much appreciate the financial support to this work from the National Natural Science Foundation of China (Grants 11172307 and11232012) and 973 Program (2014CB046200).

\section{References}

1. Sawamoto, M., Yamashita, T.: Sediment transport rate due to wave action. J. Hydrosci. Hydr. Eng. 4, 1-15 (1986)
2. Fredsøe, J., Deigaard, R.: Mechanics of Coastal Sediment Transport. World Scientific, Singapore (1992)

3. Tanaka, H., Sana, A., Yamaji, H., et al.: Experimental and numerical investigation on asymmetric oscillatory boundary layers. J. Hydrosci. Hydr. Eng. 16, 117-126 (1998)

4. Tanaka, H., Sumer, B.M., Lodahl, C.: Theoretical and experimental investigation on laminar boundary layers under cnoidal wave motion. Coast. Eng. J. 40, 81-98 (1998)

5. Carstensen, S., Sumer, B.M., Fredsøe, J.: Coherent structures in wave boundary layers. Part 1. Oscillatory motion. J. Fluid Mech. 646, 169-206 (2010)

6. Gonzalez-Rodriguez, D., Madsen, O.S.: Boundary-layer hydrodynamics and bedload sediment transport in oscillating water tunnels. J. Fluid Mech. 667, 48-84 (2011)

7. Vittori, G., Verzicco, R.: Direct simulation of transition in an oscillatory boundary layer. J. Fluid Mech. 371, 207-232 (1998)

8. Jensen, B.L., Sumer, B.M., Fredsøe, J.: Turbulent oscillatory boundary layers at high Reynolds numbers. J. Fluid Mech. 206, 265-297 (1989)

9. Sarpkaya, T.: Coherent structures in oscillatory boundary layers. J. Fluid Mech. 253, 105-140 (1993)

10. Hino, M., Kashiwayanagi, M., Nakayama, A., et al.: Experiments on the turbulence statistics and the structure of a reciprocating oscillatory flow. J. Fluid Mech. 131, 363-400 (1983)

11. Salon, S., Armenio, V., Crise, A.: A numerical investigation of the Stokes boundary layer in the turbulent regime. J. Fluid Mech. 570, 253-296 (2007)

12. Costamagna, P., Vittori, G., Blondeaux, P.: Coherent structures in oscillatory boundary layers. J. Fluid Mech. 474, 1-33 (2003)

13. Lin, P., Zhang, W.: Numerical simulation of wave-induced laminar boundary layers. Coast. Eng. 55, 400-408 (2008)

14. Lambkin, D.O., Collins, M.B., Paphitis, D.: Wave period and flow asymmetry effects on transition to turbulence in relation to sediment dynamics. J. Geophys. Res. 109, 1-10 (2004)

15. Lin, P., Li, C.W.: A $\sigma$-coordinate three-dimensional numerical model for surface wave propagation. Int. J. Numer. Meth. Fluids 38, 1045-1068 (2002)

16. Lee, S.K., Cheung, K.F.: Laminar and turbulent bottom boundary layer induced by nonlinear water waves. J. Hydraul. Eng. 126, 631644 (1999)

17. Kondo, J.: Operational Method. Baifukan, Tokyo (1956)

18. Nadaoka, K., Yagi, H., Nihei, Y., et al.: Characteristics of turbulent structure in asymmetrical oscillatory flow. Proc. Coast. Eng. 41, 141-145 (1994)

19. Nadaoka, K., Yagi, H., Nihei, Y., et al.: Turbulent structure of asymmetrical oscillatory flow. Proc. Coast. Eng. 43, 441-445 (1996)

20. Ribberink, J.S., Al-Salem, A.A.: Sheet flow and suspension of sand in oscillatory boundary layers. Coast. Eng. 25, 205-225 (1995)

21. Tanaka, H., Yamaji, H., Sana, A., et al.: A generation method of asymmetric oscillatory motion simulating cnoidal waves. Coast. Eng. J. 40, 291-306 (1998)

22. Schlatter, P., Örlü, R.: Assessment of direct numerical simulation data of turbulent boundary layers. J. Fluid Mech. 659, 116-126 (2010)

23. Spalart, P.R.: Direct simulation of a turbulent boundary layer up to $\mathrm{R}_{\theta}=1410$. J. Fluid Mech. 187, 61-98 (1988)

24. Sana, A., Tanaka, H., Yamaji, H., et al.: Hydrodynamic behavior of asymmetric oscillatory boundary layers at low Reynolds numbers. J. Hydraul. Res. 132, 1086-1096 (2006)

25. Wilcox, D.C.: Reassessment of the scale-determining equation for advanced turbulent models. AIAA J. 26, 1299-1310 (1988)

26. Menter, F.R.: Two-equation eddy-viscosity turbulence models for engineering applications. AIAA J. 32, 1598-1605 (1994)

27. Suntoyo: Study on turbulent bottom boundary layer under nonlinear waves and its application to sediment transport. [Ph.D. Thesis], Tohoku University (2006) 
28. Gayen, B., Sarkar, S., Taylor, J.R.: Large eddy simulation of a stratified boundary layer under an oscillatory current. J. Fluid Mech. 643, 233-266 (2010)

29. Radhakrishnan, S., Piomelli, U.: Large-eddy simulation of oscillating boundary layers: model comparison and validation. J. Geophys. Res. 113, 1-14 (2008)

30. Lohmann, I.P., Fredsøe, J., Sumer, B.M., et al.: Large eddy simulation of the ventilated wave boundary layer. J. Geophys. Res. 111, 1-21 (2006)
31. Piomelli, U., Balaras, E.: Wall-layer models for large-eddy simulations. Annu. Rev. Fluid Mech. 34, 349-374 (2002)

32. Piomelli, U.: Large-eddy and direct simulation of turbulent flows. Short course delivered at CFD2001-9e conference annuelle de la Societe canadienne de CFD, 19-20. Kitchener (2001)

33. Qiang, Z.: Turbulent statistics and bursting characteristics of typical wall flows. [Ph.D. Thesis], Graduate School of Chinese Academy of Sciences Doctoral (2005) 